\title{
Dynamics of stochastic non-autonomous plankton-allelopathy system
}

\author{
Xiaoqing Wen ${ }^{1}$, Hongwei Yin ${ }^{1}$ and Ying Wei ${ }^{2^{*}}$
}

"Correspondence: qzjwy@163.com ${ }^{2} S$ chool of Management, Nanchang University, Nanchang, 330031, P.R. China

Full list of author information is available at the end of the article

\begin{abstract}
In this paper, a stochastic non-autonomous plankton-allelopathy system is investigated. The existence and uniqueness of globally positive solution to this system are proved. A sufficient criterion for extinction is established. Globally asymptotical stability of this system is obtained. Some numerical simulations illustrate our main results. Finally, unavoidable conclusions are drawn.
\end{abstract}

Keywords: non-autonomous plankton-allelopathy system; stochastic perturbation; extinction; globally asymptotical stability

\section{Introduction}

In an ecological system, the plankton population is an important problem, which was first pointed out by Chattopadhyay [1]. The author in [1] built the following competitive ODE system:

$$
\left\{\begin{array}{l}
\frac{d x}{d t}=x\left(r_{1}(t)-a_{11}(t) x-a_{12}(t) y-a_{13}(t) x y\right) \\
\frac{d y}{d t}=y\left(r_{2}(t)-a_{21}(t) x-a_{22}(t) y-a_{23}(t) x y\right)
\end{array}\right.
$$

where $x, y$, respectively, denote the population density of two competing species at time $t$. $r_{1}, r_{2}$ are the intrinsic growth rates; $a_{11}, a_{22}$ are the rates of the intra-specific competition of these two species, respectively. $a_{12}, a_{21}$ are the rates of the inter-specific competition from the first and second species, respectively. $a_{13}, a_{23}$ are, respectively, the rates of the toxic inhibition of the first species by the second and vice versa. The system (1.1) assumed that each species produced a substance toxic to the other, only when the other is present. For the production of the toxic substance, allelopathy to the competing species is not instantaneous and may be delayed, and Mukhopadyay et al. in [2] studied the system (1.1) with delays. Zhen and Ma in [3] discussed the system (1.1) equipped with distributed delay and with periodically changing parameters for seasonal changes or food supplies. In addition, He et al. in [4] showed sufficient conditions to ensure the existence of almost periodic solution of the plankton-allelopathy system with impulse. However, in the real world, the spatial factor is very important, for example, diffusion of intra species from high density to low one, and cross-diffusion of inter species for chase or the presence of other species. Based on these considerations, some reaction-diffusion models of plankton allelopathy were investigated in [5-8].

(c) 2015 Wen et al. This article is distributed under the terms of the Creative Commons Attribution 4.0 International License (http://creativecommons.org/licenses/by/4.0/), which permits unrestricted use, distribution, and reproduction in any medium, provided you give appropriate credit to the original author(s) and the source, provide a link to the Creative Commons license, and indicate if changes were made. 
On the other hand, the system (1.1) of plankton allelopathy is often affected by environmental fluctuations. Thus, it is important and useful to reveal how the fluctuations affect the system (1.1). In this paper, we only examine how noises in the environment affect the growth rates in the system (1.1) with

$$
r_{i}(t) \rightarrow r_{i}(t)+\sigma_{i}(t) \dot{B}_{i}(t), \quad i=1,2,
$$

where $\left(B_{1}(t), B_{2}(t)\right)^{T}$ is a two-dimensional Brownian motion defined on a complete probability space $(\Omega, \mathcal{F}, \mathcal{P}) . \sigma_{i}^{2}, i=1,2$ denotes the intensity of the white noise. Then the system (1.1) is changed into the following form:

$$
\left\{\begin{array}{l}
d x=x\left(r_{1}(t)-a_{11}(t) x-a_{12}(t) y-a_{13}(t) x y\right) d t+\sigma_{1}(t) x d B_{1}(t) \\
d y=y\left(r_{2}(t)-a_{21}(t) x-a_{22}(t) y-a_{23}(t) x y\right) d t+\sigma_{2}(t) y d B_{2}(t) .
\end{array}\right.
$$

The initial value of the model (1.2) is $x(0)=x_{0}>0, y(0)=y_{0}>0$.

To our knowledge, the stochastic plankton-allelopathy system (1.2) has not yet been investigated. In this paper we make the first attempt to fill this gap and study some mathematical properties of (1.2), such as the existence of a global unique positive solution to (1.2), extinction, and globally asymptotical stability. The rest of the paper is organized as follows. In Section 2, the existence and uniqueness of the global positive solution to (1.2) are proved. In Section 3, sufficient conditions for the extinction of the system (1.2) are established. In Section 4, we show the globally asymptotical stability of the system (1.2). Finally, we present some numerical simulations to confirm our results.

\section{Global unique positive solution}

In this section, we will study the existence and uniqueness of global positive solution of the system (1.2). We first introduce some marks. If $f(t)$ is a continuous bounded function on $\mathbb{R}_{+}$, define

$$
f^{u}=\sup _{t \in \mathbb{R}_{+}} f(t), \quad f^{l}=\inf _{t \mathbb{R}_{+}} f(t)
$$

In this paper, we suppose that $r_{i}(t), a_{i, j}(t)$ and $\sigma_{i}(t), i=1,2, j=1,2,3$, are continuous bounded positive functions on $\mathbb{R}_{+}$. Now we show the main results of (1.2).

Lemma 2.1 There exists a unique positive local solution $(x(t), y(t))$ almost surely (a.s.) $t \in$ $\left[0, \tau_{e}\right)$ to the system (1.2) with the initial value $x_{0}, y_{0}>0$, where $\tau_{e}$ is the explosion time.

Proof Let $x(t)=\ln u(t), y(t)=\ln v(t)$. By using the Itô formula we get the following equations:

$$
\left\{\begin{array}{l}
d u=\left(r_{1}(t)-a_{11}(t) e^{u}-a_{12}(t) e^{v}-a_{13}(t) e^{u+v}-\frac{\sigma_{1}^{2}}{2}\right) d t+\sigma_{1}(t) d B_{1}(t), \\
d v=\left(r_{2}(t)-a_{21}(t) e^{u}-a_{22}(t) e^{v}-a_{23}(t) e^{u+v}-\frac{\sigma_{2}^{2}}{2}\right) d t+\sigma_{2}(t) d B_{2}(t), \\
u(0)=\ln x_{0}, \quad v(0)=\ln y_{0},
\end{array}\right.
$$

on $t \geq 0$. It is easy to see that the coefficients of (2.1) satisfy the local Lipschitz condition, and then, for any given initial values $u(0)>0, v(0)>0$, there exists a unique maximal local 
solution $u(t), v(t)$ on $\left[0, \tau_{e}\right)$, where $\tau_{e}$ is the explosion time. By the Itô formula, $x(t)=e^{u(t)}$, $y(t)=e^{\nu(t)}$ is the positive local solution to (2.1) with the initial value $x_{0}, y_{0}>0$.

Lemma 2.1 only tells us that there is a unique positive local solution model (2.1). In order to show that this solution is global, we need to show that $\tau_{e}=\infty$ a.s.

Lemma 2.2 For any given initial value $\left(x_{0}, y_{0}\right) \in \mathbb{R}_{+}^{2}$, there is a unique solution $(x(t), y(t))$ on $t \geq 0$ and the solution will remain in $\mathbb{R}_{+}^{2}$ a.s., where $\mathbb{R}_{+}^{2}=\left\{(x, y) \in \mathbb{R}_{+}^{2} \mid x, y>0\right\}$.

Proof For convenience, let

$$
\begin{aligned}
& F_{1}(x, y)=r_{1}(t)-a_{11}(t) x-a_{12}(t) y-a_{13}(t) x y, \\
& F_{2}(x, y)=r_{2}(t)-a_{21}(t) x-a_{22}(t) y-a_{23}(t) x y .
\end{aligned}
$$

The following proof is motivated by the work [9-11]. Let $n_{0}>0$ be sufficient large for $x_{0}$ and $y_{0}$ lying within the interval $\left[\frac{1}{n_{0}}, n_{0}\right]$. For each integer $n>n_{0}$, define the stopping times:

$$
\tau_{n}=\inf \left\{t \in\left[0, \tau_{e}\right]: x(t) \notin\left(\frac{1}{n}, n\right) \text { or } y(t) \notin\left(\frac{1}{n}, n\right)\right\} .
$$

Throughout this paper, we set $\inf \varnothing=\infty$. In fact, $\tau_{n}$ is increasing as $n \rightarrow \infty$. Let $\tau_{\infty}=$ $\lim _{n \rightarrow \infty} \tau_{n}$, and $\tau_{\infty} \leq \tau_{e}$ a.s. Now we only need to show $\tau_{\infty} \rightarrow \infty$. If this statement is false, there is a pair of constants $T>0$ and $\varepsilon \in(0,1)$ such that $P\left\{\tau_{\infty} \leq T\right\}>\varepsilon$. Hence, there exists an integer $n_{1} \geq n_{0}$ such that

$$
P\left\{\tau_{n} \leq T\right\} \geq \varepsilon, \quad n \geq n_{1} .
$$

We define a $C^{2}$ function $V: \mathbb{R}_{+}^{2} \rightarrow \mathbb{R}_{+}$by

$$
V(x, y)=(\sqrt{x}-1-0.5 \ln x)+(\sqrt{y}-1-0.5 \ln y) .
$$

Obviously, (2.3) is non-negative on $x, y>0$. Further, for $(x(t), y(t)) \in \mathbb{R}^{2}$ and by the Itô formula, we can obtain

$$
\begin{aligned}
d V(x, y)= & \frac{1}{2}(\sqrt{x}-1) F_{1}(x, y) d t+\frac{2-\sqrt{x}}{8} \sigma_{1}^{2}(t) d t \\
& +\frac{1}{2}(\sqrt{y}-1) F_{2}(x, y) d t+\frac{2-\sqrt{y}}{8} \sigma_{2}^{2}(t) d t \\
& +\frac{1}{2}(\sqrt{x}-1) \sigma_{1}(t) d B_{1}(t)+\frac{1}{2}(\sqrt{y}-1) \sigma_{2}(t) d B_{2}(t) .
\end{aligned}
$$

By direct calculation, we easily get

$$
\begin{aligned}
& \frac{1}{2}(\sqrt{x}-1) F_{1}(x, y)=\frac{1}{2}(\sqrt{x}-1)\left[r_{1}(t)-a_{11}(t) x-a_{12}(t) y-a_{13}(t) x y\right] \leq K_{1}, \\
& \frac{1}{2}(\sqrt{y}-1) F_{2}(x, y)=\frac{1}{2}(\sqrt{y}-1)\left[r_{2}(t)-a_{21}(t) x-a_{22}(t) y-a_{23}(t) x y\right] \leq K_{2},
\end{aligned}
$$


where $K_{1}, K_{2}$ are two some positive constants. Integrating both sides of (2.4) from 0 to $\tau_{n} \wedge T$ and then taking the expectation lead to

$$
E V\left(x\left(\tau_{n} \wedge T\right), y\left(\tau_{n} \wedge T\right)\right) \leq V\left(x_{0}, y_{0}\right)+\left(K_{1}+K_{2}\right) T .
$$

Set $\Omega_{n}=\left\{\tau_{n} \leq T\right\}$, then by (2.2) we have $P\left(\Omega_{m}\right) \geq \varepsilon$. Note that for each $\omega \in \Omega_{n}$, there is some $i$ such that $x_{i}\left(\tau_{n}, \omega\right)$ equals $n$ or $1 / n$ for $i=1,2$. Consequently, $V\left(x\left(\tau_{n} \wedge T\right), y\left(\tau_{n}, \wedge T\right)\right)$ is no less than

$$
\min \{\sqrt{n}-1-0.5 \ln n, \sqrt{1 / n}-1-0.5 \ln 1 / n\} .
$$

By (2.5) we find

$$
\begin{aligned}
V\left(x_{0}, y_{0}\right)+\left(K_{1}+K_{2}\right) T & \geq E\left[I_{\Omega_{n}}(\omega) V\left(x\left(\tau_{n}\right), y\left(\tau_{n}\right)\right)\right] \\
& \geq \varepsilon \min \{\sqrt{n}-1-0.5 \ln n, \sqrt{1 / n}-1-0.5 \ln 1 / n\},
\end{aligned}
$$

where $1_{\Omega_{n}}$ is the indicator function of $\Omega_{n}$. Letting $n \rightarrow \infty$ leads to the contraction

$$
\infty>V\left(x_{0}, y_{0}\right)+\left(K_{1}+K_{2}\right) T=\infty \text {. }
$$

We arrive at the contradiction above and complete the proof.

\section{Extinction}

In this section, we shall address the extinction of the system (1.2). The following theorem shows that the noise may induce extinction of (1.2).

Theorem 3.1 Let the conditions of $r_{1}(t)-0.5 \sigma_{1}^{2}(t)<0$ and $r_{2}(t)-0.5 \sigma_{2}^{2}(t)<0$ hold, then the population $x$ and $y$ will become extinct exponentially a.s.

Proof Define $V(x, y)=c_{1} x+c_{2} y$. By the Itô formula, we have

$$
d[\ln (x(t))]=\left(r_{1}(t)-a_{11}(t) x-a_{12}(t) y-a_{13}(t) x y-\frac{\sigma_{1}^{2}(t)}{2}\right) d t+\sigma_{1}(t) d B_{1}(t) .
$$

Integrating both sides of (3.1) leads to

$$
\begin{aligned}
\frac{\ln \left(x(t) / x_{0}\right)}{t}= & \frac{\int_{0}^{t}\left[r_{1}(t)-0.5 \sigma_{1}^{2}(s)\right] d s}{t}-\frac{\int_{0}^{t} a_{11}(t) x(s) d s}{t}-\frac{\int_{0}^{t} a_{12}(t) y(s) d s}{t} \\
& -\frac{\int_{0}^{t} a_{13}(t) x(s) y(s) d s}{t}+\frac{\int_{0}^{t} \sigma_{1}(s) d B_{1}(s)}{t} .
\end{aligned}
$$

In the same way, we can show that

$$
\begin{aligned}
\frac{\ln \left(y(t) / y_{0}\right)}{t}= & \frac{\int_{0}^{t}\left[r_{2}(t)-0.5 \sigma_{2}^{2}(s)\right] d s}{t}-\frac{\int_{0}^{t} a_{21}(t) x(s) d s}{t}-\frac{\int_{0}^{t} a_{22}(t) y(s) d s}{t} \\
& -\frac{\int_{0}^{t} a_{23}(t) x(s) y(s) d s}{t}+\frac{\int_{0}^{t} \sigma_{2}(s) d B_{2}(s)}{t} .
\end{aligned}
$$


Let $M_{i}(t)=\int_{0}^{t} \sigma_{i}(t) d B_{i}(t)$ for $i=1,2$. Then $M_{i}(t)$ is a local martingale and its quadratic variation is

$$
\left\langle M_{i}, M_{i}\right\rangle_{t}=\int_{0}^{t} \sigma_{i}^{2}(s) d s \leq\left(\sigma_{i}^{2}\right)^{u} t, \quad i=1,2 .
$$

By the strong law of large numbers for martingales [12] we have

$$
\lim _{t \rightarrow+\infty} \frac{M_{i}(t)}{t}=0 \quad \text { a.s. }
$$

From (3.2), (3.4), and $r_{1}-0.5 \sigma_{1}^{u}<0$, we have

$$
\lim _{t \rightarrow+\infty} \sup \frac{\ln (x(t))}{t} \leq \frac{\int_{0}^{t}\left[r_{1}-0.5 \sigma_{1}^{2}(s)\right] d s}{t}<0 \quad \text { a.s. }
$$

\section{Globally asymptotical stability}

In this section, we will establish a sufficient criterion for the globally asymptotical stability of the system (1.2).

Definition 4.1 The system (1.2) is said to have globally asymptotical stability if

$$
\lim _{t \rightarrow \infty}\left|x_{1}(t)-x_{2}(t)\right|=\lim _{t \rightarrow \infty}\left|y_{1}(t)-y_{2}(t)\right|=0 \quad \text { a.s. }
$$

for any two positive solutions $\left(x_{1}(t), y_{1}(t)\right)$ and $\left(x_{2}(t), y_{2}(t)\right)$ of the system (1.2).

Now, we present some important lemmas.

Lemma 4.2 Let $(x(t), y(t))$ be a solution to (1.2) with initial value $\left(x_{0}, y_{0}\right)$, then, for all $p>1$, there exist constants $L(p)$ and $G(p)$ such that

$$
E\left(x^{p}(t)\right) \leq L(p), \quad E\left(y^{p}(t)\right) \leq G(p) .
$$

Proof Define $V(x)=x^{p}$ for all $x \in \mathbb{R}_{+}, p>1$. By the Itô formula, we have

$$
d V(x)=p x^{p}\left(r_{1}(t)-a_{11}(t) x-a_{12}(t) y-a_{13}(t) x y+0.5(p-1) \sigma_{1}^{2}(t)\right) d t+p x^{p} \sigma_{1}(t) d B_{1}(t) .
$$

We use the Itô formula again with $e^{t} V(x)$, resulting in

$$
\begin{aligned}
d\left(e^{t} V(x)\right)= & p e^{t} x^{p}\left(1 / p+r_{1}(t)-a_{11}(t) x-a_{12}(t) y-a_{13}(t) x y+0.5(p-1) \sigma_{1}^{2}(t)\right) d t \\
& +p e^{t} x^{p} d B_{1}(t) .
\end{aligned}
$$

We take expectations on both sides and obtain

$$
\begin{aligned}
E\left[e^{t} x^{p}(t)\right] & =x_{0}^{p}+p E \int_{0}^{t} e^{s}\left\{x^{p}(s)\left[1 / p+r_{1}^{u}-a_{11}^{l} x-a_{12}^{l} y-a_{13}^{l} x y+0.5(p-1)\left(\sigma_{1}^{2}\right)^{u}\right]\right\} d s \\
& \leq x_{0}^{p}+p E \int_{0}^{t} e^{s}\left\{x^{p}(s)\left[1 / p+r_{1}^{u}-a_{11}^{l} x+0.5(p-1)\left(\sigma_{1}^{2}\right)^{u}\right]\right\} d s
\end{aligned}
$$




$$
\begin{aligned}
& \leq x_{0}^{p}+\int_{0}^{t} e^{s} L_{1}(p) d s \\
& =x_{0}^{p}+L_{1}(p)\left(e^{t}-1\right),
\end{aligned}
$$

where $L_{1}(p)=\frac{\left[1+r_{1}^{u} p+0.5 p(p-1)\left(\sigma_{1}^{2}\right)\right]^{p+1}}{(p+1)^{p+1} a_{11}^{l} p}$. Thus there exists a $T>0$ such that $E\left(x^{p}(x)\right) \leq 1.5 L_{1}(p)$ for all $t \geq T$. At the same time, by the continuity of $E\left(x^{p}(x)\right)$, it is well known that there exists $\tilde{L}_{1}(p)>0$ such that $E\left(x^{p}(t)\right) \leq \tilde{L}_{1}(p)$ for all $t \leq T$. Let $L(p)=\max \left\{\tilde{L}_{1}(p), 1.5 L_{1}(p)\right\}$, thus for all $t \geq 0$, we have $E\left(x^{p}(t)\right) \leq L(p)$.

On the other hand, by the same method we can see that there exists $G(p)$ such that $E\left(y^{p}(t)\right) \leq G(p)$.

Lemma 4.3 Suppose that an $n$-dimensional stochastic process $X(t)$ on $t \leq 0$ satisfies the condition

$$
E|x(t)-x(s)|^{\alpha_{1}} \leq c_{1}|t-s|^{1+\alpha_{2}}, \quad 0 \leq s, t<\infty
$$

for some positive constants $\alpha_{1}, \alpha_{2}$, and $c$. Then there exists a continuous modification $\tilde{x}(t)$ of $x(t)$ which has the property that, for every $\theta \in\left(0, \alpha_{2} / \alpha_{1}\right)$, there is a positive random variable $h(\omega)$ such that

$$
P\left\{\omega: \sup _{0<|t-s|<h(\omega), 0<s, t<\infty} \frac{\tilde{x}(t, \omega)-x(t, \omega)}{|t-s|^{\theta}} \leq \frac{2}{1-2^{-\theta}}\right\}=1 .
$$

In other words, almost every sample path of $\tilde{x}(t)$ is locally but uniformly Hölder continuous with exponent $\theta$.

Lemma 4.4 Let $(x(t), y(t))$ be a solution of (1.2) on $t \geq 0$, then almost every sample path of $x(t), y(t)$ is uniformly continuous.

Proof The first equation of the system (1.2) is equivalent to the following stochastic integral equation:

$$
x(t)=x_{0}+\int_{0}^{t} x\left(r_{1}(s)-a_{11}(s) x-a_{12}(s) y-a_{13}(s) x y\right) d s+\int_{0}^{t} \sigma_{1} x d B_{1}(s) .
$$

By Lemma 4.2 and Young's inequality,

$$
\begin{aligned}
E \mid & \left.x\left(r_{1}(t)-a_{11}(t) x-a_{12}(t) y-a_{13}(t) x y\right)\right|^{p} \\
& \leq 0.5 E|x|^{2 p}+0.5 E\left|r_{1}(t)-a_{11}(t) x-a_{12}(t) y-a_{13}(t) x y\right|^{2 p} \\
& \leq 0.5\left\{L(2 p)+4^{2 p-1}\left[\left(\left|r_{1}\right|^{u}\right)^{2 p}+a_{11}^{u} L(2 p)+a_{12}^{u} G(2 p)+a_{13}^{u} E|x y|^{2 p}\right]\right\} \\
& \leq 0.5\left\{L(2 p)+4^{2 p-1}\left[\left(\left|r_{1}\right|^{u}\right)^{2 p}+a_{11}^{u} L(2 p)+a_{12}^{u} G(2 p)+0.5 a_{13}^{u} L(4 p)+0.5 a_{13}^{u} G(4 p)\right]\right\} \\
& :=K_{2}(p) .
\end{aligned}
$$

Moreover, by the help of the moment inequality for stochastic integrals, it follows that, for $0 \leq t_{1} \leq t_{2}$ and $p>2$,

$$
E\left|\int_{t_{1}}^{t_{2}} \sigma_{1}(s) x(s) d B_{1}(s)\right|^{p} \leq\left[\left(\sigma_{1}^{2}\right)^{u}\right]^{p}\left[\frac{p(p-1)}{2}\right]^{p / 2}\left(t_{2}-t_{1}\right)^{p / 2} L(p) .
$$


Therefore, for $0<t_{1}<t_{2}<\infty, t_{2}-t_{1} \leq 1,1 / p+1 / q=1$, we have

$$
\begin{aligned}
& E\left(\left|x_{2}(t)-x_{1}(t)\right|^{p}\right) \\
& =E\left|\int_{t_{1}}^{t_{2}} x\left(r_{1}(t)-a_{11}(t) x-a_{12}(t) y-a_{13}(t) x y\right) d s+\int_{t_{1}}^{t_{2}} \sigma_{1}(s) x(s) d B_{1}(s)\right|^{p} \\
& \leq 2^{p-1} E\left|\int_{t_{1}}^{t_{2}} x\left(r_{1}(t)-a_{11}(t) x-a_{12}(t) y-a_{13}(t) x y\right) d s\right|^{p} \\
& \quad+2^{p-1} E\left|\int_{t_{1}}^{t_{2}} \sigma_{1}(s) x(s) d B_{1}(s)\right|^{p} \\
& \quad \leq 2^{p-1}\left(t_{2}-t_{1}\right)^{p / q+1} K_{2}(p)+2^{p-1}\left[\left(\sigma_{1}^{2}\right)^{u}\right]^{p}\left[\frac{p(p-1)}{2}\right]^{p / 2}\left(t_{2}-t_{1}\right)^{p / 2} L(p) .
\end{aligned}
$$

Let $K_{3}(p)=\max \left\{K_{2}(p),\left(\sigma_{1}^{2}\right)^{p} L(p)\right\}$, then one obtains

$$
E\left(\left|x_{2}(t)-x_{1}(t)\right|^{p}\right) \leq 2^{p-1}\left(t_{2}-t_{1}\right)^{p / 2}\left[1+\left(\frac{p(p-1)}{2}\right)^{p / 2}\right] K_{3}(p)
$$

From Lemma 4.3 it follows that almost every path of $x(t)$ is locally but uniformly Hölder continuous with exponent $\theta \in\left(0, \frac{p-2}{2 p}\right)$ and therefore almost every sample path of $x(t)$ is uniformly continuous on $t \geq 0$. In the same way, we can check that almost every path of $y(t)$ is uniformly continuous.

Lemma 4.5 [13] Let $f$ be a non-negative function on $\mathbb{R}_{+}$such that $f$ is integrable and is uniformly continuous. Then $\lim _{t \rightarrow \infty} f(t)=0$.

Theorem 4.6 The system (1.2) is globally asymptotically stable.

Proof We introduce the following function:

$$
V(t)=\left|\ln x_{1}(t)-\ln x_{2}(t)\right|+\left|\ln y_{1}(t)-\ln y_{2}(t)\right|,
$$

then the function $V(t)$ is continuous and positive function on $t \geq 0$. We directly calculate and use the Itô formula to find

$$
\begin{aligned}
d^{+} V(x)= & \operatorname{sgn}\left(x_{1}-x_{2}\right)\left[-a_{11}\left(x_{1}-x_{2}\right)-a_{12}\left(y_{1}-y_{2}\right)-a_{13}\left(x_{1} y_{1}-x_{2} y_{2}\right)\right] d t \\
& +\operatorname{sgn}\left(y_{1}-y_{2}\right)\left[-a_{21}\left(x_{1}-x_{2}\right)-a_{22}\left(y_{1}-y_{2}\right)-a_{23}\left(x_{1} y_{1}-x_{2} y_{2}\right)\right] d t
\end{aligned}
$$

Integrating both sides leads to

$$
\begin{aligned}
V(t) \leq & V(0)+\int_{0}^{t}\left[-a_{11}\left|x_{1}-x_{2}\right|+a_{12}\left|y_{1}-y_{2}\right|+a_{13}\left|x_{1} y_{1}-x_{2} y_{2}\right|\right] d s \\
& +\int_{0}^{t}\left[a_{21}\left|x_{1}-x_{2}\right|-a_{12}\left|y_{1}-y_{2}\right|+a_{23}\left|x_{1} y_{1}-x_{2} y_{2}\right|\right] d s
\end{aligned}
$$


Figure 1 Existence of the solution to the system (1.2). The parameters are set as $r_{1}=0.1+0.01 \sin t$, $r_{2}=0.1+0.05 \sin t, a_{11}=a_{22}=0.1+0.01 \sin t$, $a_{21}=a_{21}=0.22+0.02 \sin t, a_{13}=a_{23}=0.1+0.01 \sin t$ and $\sigma_{1}^{2}=\sigma_{2}^{2}=0.4+0.04 \sin t$.

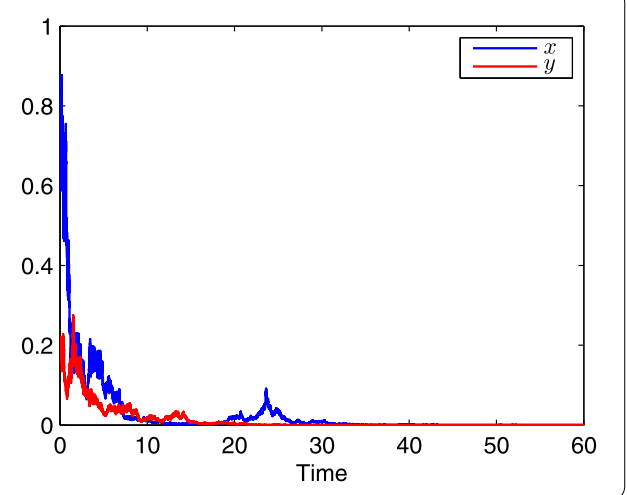

then

$$
\begin{aligned}
V(t) & -\int_{0}^{t}\left[-a_{11}\left|x_{1}-x_{2}\right|+a_{12}\left|y_{1}-y_{2}\right|+a_{13}\left|x_{1} y_{1}-x_{2} y_{2}\right|\right] d s \\
& -\int_{0}^{t}\left[a_{21}\left|x_{1}-x_{2}\right|-a_{12}\left|y_{1}-y_{2}\right|+a_{23}\left|x_{1} y_{1}-x_{2} y_{2}\right|\right] d s \leq V(0)<\infty .
\end{aligned}
$$

Thus from $V(t) \geq 0$ one gets

$$
\left|x_{1}(t)-x_{2}(t)\right| \in L^{1}[0, \infty), \quad\left|y_{1}(t)-y_{2}(t)\right| \in L^{1}[0, \infty) .
$$

In view of Lemmas 4.4 and 4.5 , the desired assertion is obtained.

\section{Numerical simulations}

In this section, we will show some figures to illustrate our main theorems by using the Milstein method [14]. For the system (1.2), we consider the following discretized equation:

$$
\begin{aligned}
x_{k+1}-x_{k}= & x_{k}\left[r_{1}(k \Delta t)-a_{11}(k \Delta t) x_{k}-a_{12}(k \Delta t) y_{k}-a_{13}(k \Delta t) x_{k} y_{k}\right] \Delta t \\
& +\sigma_{1}(k \Delta t) x_{k} \sqrt{\Delta t} \xi_{k}-0.5 \sigma_{1}^{2}(k \Delta t) x_{k}\left(\xi_{k}^{2}-1\right) \Delta t, \\
y_{k+1}-y_{k}= & y_{k}\left[r_{2}(k \Delta t)-a_{21}(k \Delta t) x_{k}-a_{22}(k \Delta t) y_{k}-a_{23}(k \Delta t) x_{k} y_{k}\right] \Delta t \\
& +\sigma_{2}(k \Delta t) y_{k} \sqrt{\Delta t} \eta_{k}-0.5 \sigma_{2}^{2}(k \Delta t) y_{k}\left(\eta_{k}^{2}-1\right) \Delta t,
\end{aligned}
$$

where $\xi_{k}$ and $\eta_{k}, k=1,2, \ldots, n$ are Gaussian random variables which follow the standard normal distribution.

Set the initial value $\left(x_{0}, y_{0}\right)=(0.6,0.2)$. Let $r_{1}=0.1+0.01 \sin t, r_{2}=0.1+0.05 \sin t, a_{11}=$ $a_{22}=0.1+0.01 \sin t, a_{21}=a_{21}=0.22+0.02 \sin t$, and $a_{13}=a_{23}=0.1+0.01 \sin t$. We choose $\sigma_{1}^{2}=\sigma_{2}^{2}=0.4+0.04 \sin t$, then $r_{1}-0.5 \sigma_{1}^{2}<0, r_{2}-0.5 \sigma_{2}^{2}<0$. Furthermore, Theorem 3.1 implies the extinction of both $x, y$. Figure 1 confirms this result.

In Figure 2, we only change the noise intensities, that is, $\sigma_{1}=0.1+0.04 \sin t, \sigma_{2}=0.2+$ $0.02 \sin t$, and the other parameters are still fixed as in Figure 1. From Theorem 4.6, we know that the system (1.2) has globally asymptotical stability. Figure 2 illustrates this result. 

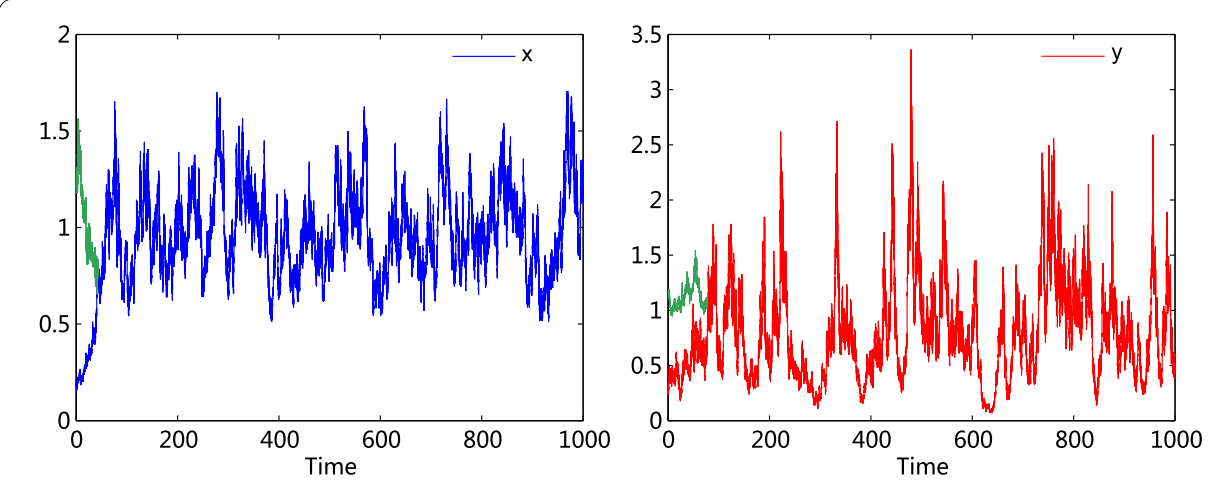

Figure 2 Globally asymptotical stability of the system (1.2). The noise intensities are set as $\sigma_{1}=0.1+0.04 \sin t, \sigma_{2}=0.2+0.02 \sin t$. The other parameters are as in Figure 1.

\section{Conclusion}

This paper has studied the existence and uniqueness of the positive solution for a stochastic non-autonomous plankton-allelopathy system. Moreover, we have obtained the sufficient conditions of extinction for this system. Furthermore, the globally asymptotical stability of this system has been proved in this paper. Finally, we illustrate some results by numerical simulations for some periodic parameters. These results are useful because the extinction thresholds are very important for the system of species. In further investigation, we may consider some more realistic and complex models such as incorporating spatial diffusion in this stochastic system. The spatial factors can affect the dynamical properties of the stochastic plankton-allelopathy system.

Competing interests

The authors declare that they have no competing interests.

Authors' contributions

XW carried out the main parts of the draft. HY and YW provided the main idea of this paper. All authors read and approved the final manuscript

\section{Author details}

${ }^{1}$ School of Science, Nanchang University, Nanchang, 330031, P.R. China. ${ }^{2}$ School of Management, Nanchang University, Nanchang, 330031, P.R. China.

\section{Acknowledgements}

The authors would like to thank the anonymous referees for their carefulness and helpful comments, which greatly improved this paper. This work was supported by the NSF of Jiangxi (No. 20151BAB212011).

Received: 17 November 2014 Accepted: 10 July 2015 Published online: 21 October 2015

\section{References}

1. Chattopadhyay, J: Effect of toxic substances on a two-species competitive system. Ecol. Model. 84, 287-289 (1996)

2. Mukhopadhyay, A, Chattopadhyay, J, Tapaswi, PK: A delay differential equations model of plankton allelopathy. Math. Biosci. 149, 167-189 (1998)

3. Zhen, J, Ma, Z: Periodic solutions for delay differential equations model of plankton allelopathy. Comput. Math. Appl. 44, 491-500 (2002)

4. $\mathrm{He}, \mathrm{M}, \mathrm{Chen}, \mathrm{F}, \mathrm{Li}, \mathrm{Z}$ : Almost periodic solution of an impulsive differential equation model of plankton allelopathy. Nonlinear Anal., Real World Appl. 11, 2296-2301 (2010)

5. Jia, Y, Wu, J, Xu, HK: Spatial pattern in a ecosystem of phytoplankton-nutrient from remote sensing. J. Math. Anal. Appl. 402, 23-34 (2013)

6. Li, Z, Chen, FD, He, MX: Asymptotic behavior of the reaction-diffusion model of plankton allelopathy with nonlocal delays. Nonlinear Anal., Real World Appl. 12, 1748-1758 (2011)

7. Tian, C, Zhang, L: Delay-driven irregular spatiotemporal patterns in a plankton system. Phys. Rev. E 88, 012713 (2013)

8. $\mathrm{Du}, \mathrm{YH}, \mathrm{Hsu}, \mathrm{SB}$ : On a nonlocal reaction-diffusion problem arising from the modeling of phytoplankton growth. SIAM J. Math. Anal. 42, 1305-1333 (2010) 
9. Du, B: Existence, extinction and global asymptotical stability of a stochastic predator-prey model with mutual interference. J. Appl. Math. Comput. 46, 79-91 (2014)

10. Liu, M, Wang, K: Global stability of a nonlinear stochastic predator-prey system with Beddington-Deangelis functional response. Commun. Nonlinear Sci. Numer. Simul. 16, 1114-1121 (2011)

11. Mao, X, Li, X: Population dynamical behavior of non-autonomous Lotka-Volterra competitive system with random perturbation. Discrete Contin. Dyn. Syst. 24, 523-545 (2009)

12. Watanabe, S, Ikeda, N: Stochastic Differential Equations and Diffusion Processes. Elsevier, Amsterdam (1981)

13. Barbalat, I: Systems d'équations différential d'oscillations nonlinéaires. Rev. Roum. Math. Pures Appl. 4, 267-270 (1959)

14. Higham, DJ: An algorithmic introduction to numerical simulation of stochastic differential equations. SIAM Rev. 43, 525-546 (2001)

Submit your manuscript to a SpringerOpen ${ }^{\circ}$ journal and benefit from:

- Convenient online submission

Rigorous peer review

- Immediate publication on acceptance

- Open access: articles freely available online

- High visibility within the field

- Retaining the copyright to your article 